\title{
La formación ciudadana en el contexto del posacuerdo en Colombia: experiencias en la educación media
}

\section{citizen education in the context of the post-agreement in Colombia: experiences in secondary education}

\author{
Sandra Gutiérrez Abella ${ }^{1}$ \\ Yenny Marcela Sánchez Aubio² \\ Mauricio Zabala Hernández ${ }^{3}$ \\ Brenda González Cuéllar"
}

(-)

Recepción: 03/07/2020
().

Aprobación: 24/08/2020
-

Publicación: 18/12/2020

\section{Para citar este artículo:}

Gutiérrez Abella, S., Sánchez Rubio, Y. M., Zabala Hernández, M., \& González

Cuéllar, B. (2020). La formación ciudadana en el contexto del posacuerdo en Colombia: experiencias en la educación media. Indagare, (8), 22-29.

https://doi.org/10.35707/indagare/802



\footnotetext{
${ }^{1}$ Grupo de investigación GESE. Universidad de Ibagué, Colombia. ORCID: 0000-0003-1090-9886. Correo electrónico: sandra.gutierrez@unibague.edu.co

${ }^{2}$ Grupo de investigación GESE. Universidad de Ibagué, Colombia. ORCID: 0000-0002-8634-2030. Correo electrónico: yenny.sanchez@unibague.edu.co

${ }^{3}$ Grupo de investigación EUlogos. Universidad de Ibagué, Colombia. ORCID: 0000-0002-3696-1781. Correo electrónico: mauricio.zabala@unibague.edu.co

${ }^{4}$ Grupo de investigación EULOGOS. Universidad de Ibagué, Colombia. ORCID: 0000-0002-2216-9451. Correo electrónico: brenda.gonzalez@unibague.edu.co
} 


\title{
Resumen
}

Comprender los procesos de formación ciudadana y para la paz que se desarrollan en el contexto escolar, constituye un elemento importante a la hora de fortalecer una educación de calidad que potencie la convivencia, la armonía y el respeto hacia el otro en el contexto del posacuerdo. El presente artículo refleja una apuesta por la integración de espacios de reflexión y resolución de conflictos, en los que juegan un papel fundamental los actores de la escuela: estudiantes, profesores, directivos y padres de familia.

\begin{abstract}
Understanding the processes of civic education and for peace that take place in the school context, represents an important element when it comes to strengthening quality education that promotes coexistence, harmony and respect for others in the context of the post-agreement. This article reflects a commitment to the integration of spaces for reflection and conflict resolution, in which school actors-students, teachers, directors, and parents-play a fundamental role.
\end{abstract}

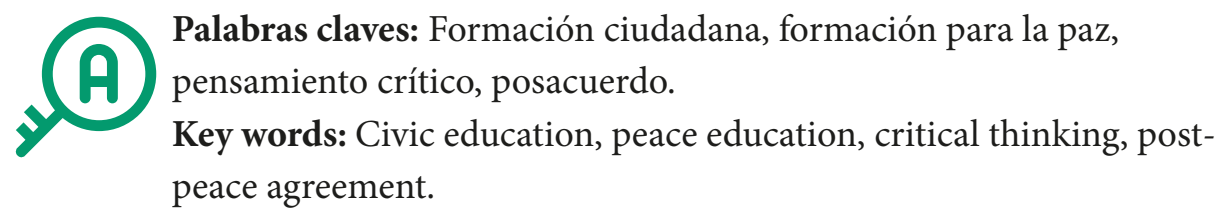

\section{Introducción}

Educar es una tarea fundamental en cualquier sociedad. En Colombia particularmente hay una preocupación por formar para la paz en un contexto de posacuerdo, como resultado de la firma del acuerdo de paz con las FARC y el inicio de su implementación en el año 2017. La expectativa y tránsito por el que pasan los ciudadanos se asocia a la reflexión que deben hacer frente a las fases pactadas, el reconocimiento de la información y la incidencia de su papel en todo el proceso. Pero construir paz también implica reconocer que los colombianos hacen parte del proceso; niños, adolescentes y adultos deben aprovechar este espacio para comprender sus derechos y participar democráticamente.

En este mismo sentido, es primordial formar en la resolución de conflictos y contribuir a una cultura de paz a través de prácticas de enseñanza y aprendizaje; ejemplo de ello son las Cátedras de Paz promovidas por el Ministerio de Educación Nacional, lo cual supone su reconocimiento e implementación en las instituciones educativas, la revisión de las prácticas pedagógicas y el compromiso por garantizar y promover 
espacios para que los miembros de la comunidad educativa puedan participar activa y propositivamente. Significa también considerar las formas en las que los sujetos que hacen parte de los procesos de enseñanza y aprendizaje, afrontan los cambios sociales y políticos que supone el posacuerdo y dan una mirada a las transformaciones sociales y al recorrido histórico del país.

Por todo lo anterior, con esta investigación se pretenden identificar las estrategias, incluidas las Cátedras de Paz, que fortalecen y aportan al desarrollo de los procesos educativos en el contexto del posacuerdo; tal como diría Hoyos, citado por Pineda (2014): "El proceso educativo es una relación necesaria entre la tradición y lo nuevo" (p. 166). Es preciso reconocer los esfuerzos que las instituciones educativas realizan para fortalecer espacios de convivencia a través de la formación de estudiantes críticos, analíticos, capaces de convivir en distintos entornos sociales, de entender y asumir todos los acontecimientos de una época marcada por la violencia y el conflicto. En suma, comprender y valorar las estrategias que se desarrollan desde las instituciones educativas, a partir de vivencias, necesidades del contexto, actitudes y propuestas de estudiantes, profesores, directivos y padres de familia.

Para estos propósitos, es fundamental el papel que el docente desempeña al tratar de generar espacios democráticos dentro de las aulas, lograr que sus estudiantes entiendan que su participación es necesaria e importante, y que esta requiere de una mirada crítica que les amplíe sus horizontes; en últimas, un docente que los motive a participar de los cambios sociales y políticos del país. Entendido esto, más allá de esquematizar la violencia y las situaciones problemáticas que ha traído el conflicto, se busca identificar espacios resilientes, en los que se privilegia el diálogo, el consenso y el disenso, el respeto de los derechos humanos, la paz y la justicia, entre otros.

Con lo anterior, la escuela debe garantizar espacios de formación ciudadana para que el estudiante se integre a las políticas de acción del proceso de paz, es necesario efectuar una revisión de las prácticas y acciones que se llevan a cabo al interior de las instituciones educativas para ver cómo impactan o qué tan efectivas pueden ser estas, a la hora de abordar situaciones de violencia y conflicto; es decir, reconocer los espacios en los que docentes y estudiantes intercambian diálogos, emociones y experiencias, en un contexto en el que se promueve la cultura por la democracia, el reconocimiento de la alteridad y la preocupación por el bien común.

En el proceso resulta fundamental aproximarse a las actividades o metodologías que realiza la comunidad educativa, con el fin de potenciar la convivencia, la armonía y el respeto hacia el otro, garantizando una educación de calidad que se erija sobre las distintas realidades a las que se enfrentan los ciudadanos todos los días. Para ello, es necesario 
revisar las directrices dadas por el Ministerio de Educación en materia de formación para la paz y de qué forma estas se han implementado en los centros educativos; a su vez, visibilizar las maneras a través de las cuales las instituciones educativas han incorporado estas normativas dentro de su currículo; por otro lado, es primordial reconocer otras acciones que emergen de modo espontáneo por parte de la comunidad educativa y que igualmente se enmarcan en la apuesta de fortalecer la formación ciudadana. Asimismo, es necesario reconocer las ideas que profesores, estudiantes, directivos y padres de familia tienen frente a lo que ha sido el conflicto armado en Colombia, y a la manera en que las instituciones educativas han asumido su responsabilidad desde los distintos espacios de formación. Por último, se busca promover buenas prácticas de enseñanza y aprendizaje que fortalezcan el quehacer docente y repercutan en una educación de calidad, responsable, crítica e integral.

En otras palabras, el trabajo para realizar se traduce como una apuesta a la integración de espacios de reflexión y participación para formar en ciudadanía. En todo esto juega un papel fundamental la escuela, que de manera transversal invita a los miembros de la comunidad a trabajar de manera articulada, para integrar al currículo el pensamiento crítico y el empoderamiento de sus estudiantes hacia la resolución de conflictos desde una actitud participativa; de manera que tanto estudiantes como profesores entiendan el valor de discutir, argumentar y utilizar el lenguaje como espacio para construir una sociedad democrática.

\section{Materiales y métodos}

La presente propuesta de investigación se encuentra enmarcada en el paradigma cualitativo, por cuanto busca realizar una aproximación a la comprensión de fenómenos sociales y la manera en que estos se manifiestan al interior de comunidades e individuos. En términos generales, la investigación cualitativa adquiere sentido en la medida en que interpreta contextos determinados, sin partir de modelos predeterminados, a través de la interacción asertiva entre investigador e investigado, por medio de técnicas e instrumentos sistemáticos, que permiten recoger la información necesaria con la cual describir, comprender e interpretar una realidad particular.

La investigación cualitativa se define también como un proceso naturalista, en tanto no recrea artificialmente los entornos que estudia, es interpretativa, sistemática, contextual, y, además, es un "campo [...] inherentemente político [...] construido por múltiples posiciones éticas y políticas" (Denzin y Lincoln, 1994, citados por Guardián, 2007, p. 34). De allí que sea constante su búsqueda por comprender e interpretar lo propio, lo particular, lo subjetivo, lo simbólico, lo íntimo, lo profundo y cómo ello permite estructurar y definir realidades sociales. 
Este proceso es además continuo y progresivo, dado que no se limita a la confirmación o refutación de hipótesis estáticas y más bien articula la teoría, la interacción de los actores y la información en un contexto determinado, con el propósito de ir estructurando intersubjetivamente diferentes realidades socioculturales. Esto se evidencia en la flexibilidad del diseño, lo cual supone un ejercicio constante de identificación de hipótesis o supuestos que emergen y otorgan sentido a la interpretación. De acuerdo con Taylor y Bogdan (1992), citados por Guardián (2007), existen otras características propias de la investigación cualitativa: es inductiva, holística, interactiva y reflexiva, naturalista, libre, abierta, humanista y rigurosa.

Figura 1. Actividades realizadas en el marco de los espacios de formación ciudadana

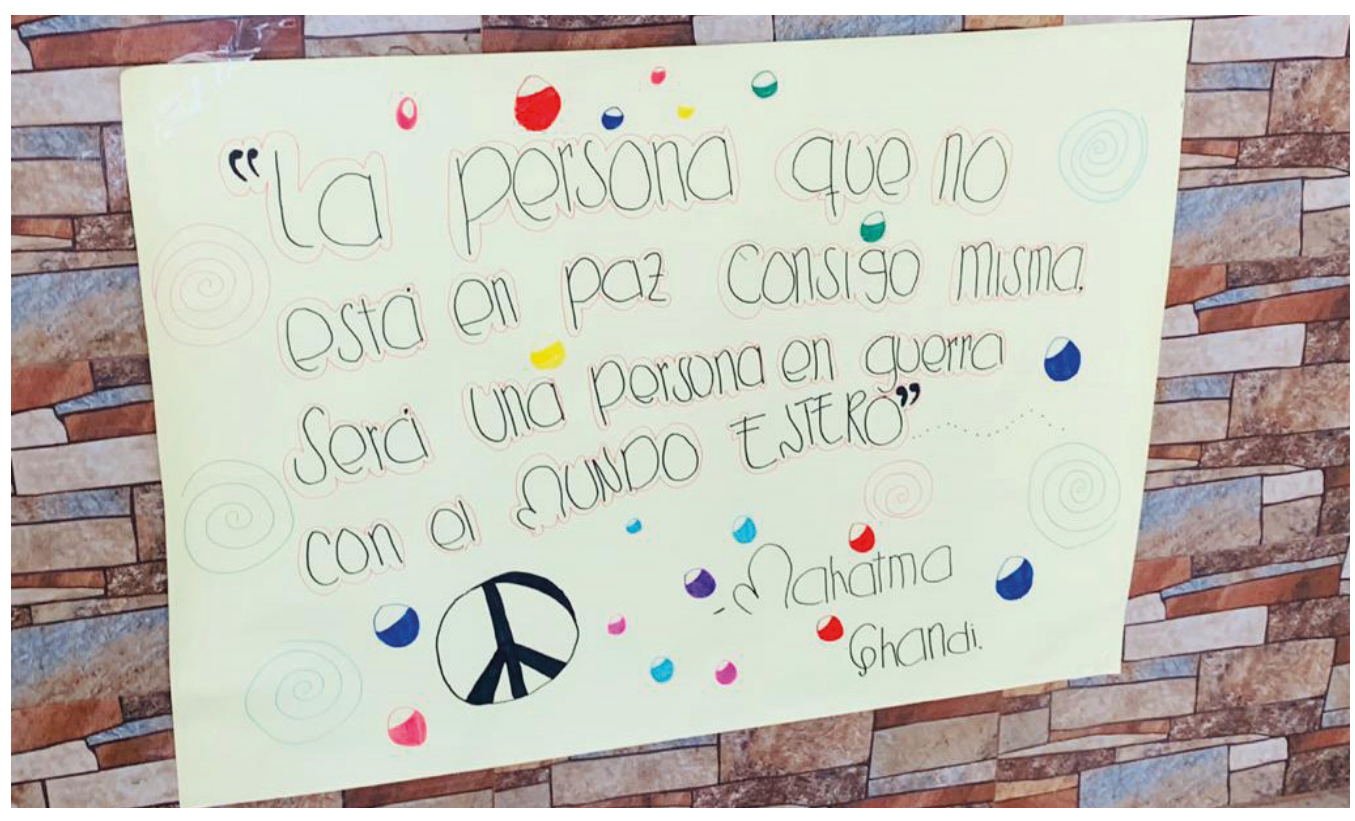

Fuente: autores

Si bien la perspectiva cualitativa es amplia, se entiende el método como un protocolo o procedimiento a través del cual el investigador se aproxima sistemática y creativamente a un objeto de conocimiento determinado. Para Mora (2010):

El proceder del investigador puede referir con exclusividad a un método, o actuar a través de la combinación o integración de diversos métodos - o diversas técnicas-, esto, con arreglo a las problemáticas y objetivos de investigación que establezca, como también a los contextos en los cuales desarrolle sus estudios. (Mora, 2010, p. 13). 
INDAGA]ZE e-ISSN: 2357-5042 • Número 8 (2020) • Universidad de Ibagué • doi: https://doi.org/10.35707/indagare/802

En tanto la presente propuesta tiene por objeto describir y comprender contextos sociales particulares, el método empleado será el etnográfico. Atkinson y Hammersley (1994), citados por Guardián (2007), identifican la etnografía como una forma de investigación social caracterizada por los siguientes rasgos:

a) Un fuerte énfasis en la exploración de la naturaleza particular de los fenómenos socioeducativos, más que en llevar a cabo pruebas de hipótesis acerca de ellos.

b) Una tendencia a trabajar con datos primarios 'no estructurados', es decir, datos que no se han codificado previamente a su recolección en un conjunto de categorías analíticas cerradas.

c) Una investigación de un número pequeño de casos, a veces solo un caso, pero en detalle.

d) Un análisis de datos que involucra la interpretación explícita de los significados y funciones de las acciones humanas, producto que toma la forma de descripciones y explicaciones principalmente verbales. (Guardián, 2007, pp. 93-94).

Como resulta imposible comprender o interpretar una realidad determinada sin una mirada contextual e histórica, el método etnográfico exige armar un protocolo que posibilite tejer relaciones cercanas entre investigador e investigado, en un medio social o institucional determinado, mediante técnicas o procedimientos bien definidos a través de los cuales se recoja, codifique, analice y comprenda, la información obtenida para que sea concordante con los objetivos establecidos.

La metodología, por su parte, comprende el cómo; es decir, los medios, los procedimientos concretos para recoger la información, además de la elección de las técnicas e instrumentos con los que se ha de recolectar, y también los mecanismos de análisis de los datos. La presente propuesta de investigación se desarrollará en cuatro etapas simultáneas o sucesivas, en consonancia con el paradigma cualitativo. Estas se han clasificado de acuerdo con el tipo de información que se espera obtener, a los objetivos y a la manera de interpretar los resultados, así: documental, etnográfica, de análisis y aplicada.

La etapa documental hace referencia a la revisión de documentos institucionales y normativos que abordan la Cátedra de Paz, los estándares de formación ciudadana del Ministerio de Educación Nacional (MEN) y el Proyecto Educativo Institucional (PEI) de las instituciones educativas para identificar cómo se implementan las directrices del Gobierno en el currículo. 
La etapa etnográfica involucra el diseño e implementación de todos los instrumentos a través de los cuales se recoge la información necesaria para reconocer y comprender las percepciones de los actores de toda la comunidad educativa; entre estos se destacan las entrevistas, las encuestas, los grupos focales y las observaciones de todo tipo de escenarios que involucren la formación ciudadana y para la paz.

La etapa de análisis constituye el eje principal del proyecto, toda vez que integra las voces de los distintos miembros que conforman la comunidad educativa y las contrasta con las directrices del Ministerio de Educación Nacional, con el currículo de la institución y con el punto de vista de los expertos, todo ello con el fin de conocer y comprender la coherencia o la disparidad que existe entre aquello que se dice y se hace, qué se hace y se piensa, qué se hace al interior de la escuela.

En la etapa aplicada, se propone el diseño e implementación de algunos talleres para promover procesos de enseñanza y aprendizaje sobre formación ciudadana y para la paz, orientadas a algunos profesores de las instituciones educativas en las que se desarrollará el proyecto.

\section{Resultados esperados}

A través del desarrollo del proyecto, y al tener en cuenta que aún se encuentra en ejecución, se esperan lograr los siguientes resultados:

- Consolidar espacios significativos de formación ciudadana y para la paz entre los miembros de la comunidad académica.

- Identificar estrategias que se desarrollen en las instituciones educativas, diferentes a las propuestas por el Ministerio de Educación Nacional, que se encuentren orientadas a la formación ciudadana y para la paz.

- Vincular a los padres de familia a los propósitos de formación ciudadana y para la paz, que promueve la institución educativa; de manera que logre impactar los hogares y la vida fuera de la escuela.

- Lograr que los estudiantes de todos los niveles interioricen y participen de las acciones de formación ciudadana promovidas en las instituciones y las incorporen en su vida cotidiana.

- Lograr que los hallazgos que se deriven de este proyecto sirvan como insumo para orientar políticas en materia de educación en el contexto del posacuerdo.

- Lograr que en las instituciones educativas se generen espacios para que se lleven a cabo acciones puntuales que contribuyan a la formación ciudadana y para la paz (comités de convivencia, grupos de resolución de conflictos entre estudiantes, consultorios de paz). 
INDAGA]EE e-ISSN: 2357-5042 • Número 8 (2020) • Universidad de Ibagué • doi: https://doi.org/10.35707/indagare/802

- Configurar espacios de formación en los que los profesores se integren y de manera colaborativa compartan experiencias e ideas alrededor de la formación ciudadana y para la paz, que sean eje transversal en su quehacer pedagógico.

- Caracterizar la manera cómo se implementa la Cátedra de Paz en las instituciones educativas.

\section{Ficha técnica del proyecto}

Título del proyecto: La lectura y la escritura como estrategias de formación ciudadana en algunas instituciones educativas de Ibagué en un contexto de posacuerdo.

PRIT: Educación y bienestar para el desarrollo humano integral.

Código del proyecto: $19-492-$ INT.

Palabras claves: Formación ciudadana, formación para la paz, pensamiento crítico, posacuerdo.

Grupo de investigación: GESE y EULOGOS.

Investigador principal: Sandra Gutiérrez Abella.

Correo electrónico: sandra.gutierrez@unibague.edu.co

\section{Referencias}

Guardián Fernández, A. (2007). El paradigma cualitativo en la investigación socio-educativa. San José, Costa Rica: Colección IDER. Coordinación Educativa y Cultural Centroamericana (CECC) Agencia Española de Cooperación Internacional (AECI). Recuperado de https://bit.ly/2KG60PG

Mora Nawrath, H.I. (2010). El método etnográfico: Origen y fundamentos de una aproximación multitécnica. Forum: Qualitative Social Research, 11(2), 1-31. http://dx.doi.org/10.17169/fqs11.2.1283

Pineda-R., D. A. (2014). Guillermo Hoyos-Vásquez: La formación de ciudadanos en la construcción de la democracia en Colombia. Magis, Revista Internacional de Investigación en Educación, 6 (13), 161172. https://doi.org/10.11144/Javeriana.m6-13.ghfcw 